\title{
Moving Forward as a Growing Platform of Geriatric Medicine and Gerontologic Research
}

\author{
Jae-Young Lim \\ Department of Rehabilitation Medicine, Seoul National University Bundang Hospital, Seoul National University College of Medicine, Seoul, Korea
}

For the past 20 years, Annals of Geriatric Medicine and Research (AGMR) has played a significant role in the development of academic research in the field of geriatric medicine as the official journal of the Korean Geriatrics Society. The number of cross-reference and total citations has markedly increased since AGMR was listed in the Emerging Sources Citation Index database in March 2018. As the name AGMR implies, the journal's comprehensive aim and scope cover not only clinical studies of geriatric medicine but basic science, pre-clinical, and translational studies in the field of gerontology. Notably, AGMR also offers future perspectives on geriatric issues and emerging research needs in Asian countries faced with a rapidly growing aging population.

We are excited to announce that the Korean Society for Gerontology, an academic group of representative scientists in aging research, has endorsed AGMR as their official journal. The Korean Society for Gerontology was founded in 1989 by scientists and researchers with an interest and enthusiasm to understand the fundamental principles of the life phenomenon of aging and contribute to the healthy lives of aging people. It has become a professional academic organization representing aging research in Korea. The society, which celebrates its 30th anniversary this year, is making great efforts to strengthen their members' research capabilities and expand exchanges at various levels with international academic organizations - all to make a leap forward in aging research. In a previous issue, cellular $\mathrm{NAD}^{+}$levels were highlighted as a key determinant of mitochondrial quality in aging and degenerative diseases as reported by Hwang and Hwang. ${ }^{1)}$ This distinguished review article regarding mitochondrial biogenesis is a good example of gerontologic basic research featured in AGMR. In the current issue, Kwak and Kwon ${ }^{2)}$ have presented the current status of drug development in sarcopenia. The field of drug development for sarcopenia is a new arena of future medical and biological research among health care professionals and scientists. The endorsement as the official journal of the two leading academic societies in geriatric medicine and gerontologic research is expected to expand the readership of AGMR and to promote quantitative and qualitative growth of the academic contents of the journal. More qualified research outcomes and products will be targeted for publication in AGMR. Editorial experts from the two societies will contribute to the development of the journal by participating in editorial and reviewer boards.

A second exciting development is the launch of the new journal website. Overall, the design and visualization of the website was improved to make it more user-friendly. Now, it is easier to browse through the website and find articles quickly by sorting via article category. In addition, the function of browsing through the articles has been enhanced so that the readers can see the current status of the articles being read and cited at a glance with the metric information of the journal such as journal hits, downloads, and cross-reference citations. At the same time, we have also launched a mobile-friendly application version, which enables users to communicate with each other using social network services. It will be intriguing to see what the journal has created and where the future will reach.

It is our hope that AGMR will move forward as a growing platform for academic needs of professionals and researchers in geriatrics and gerontology and will aid in dissemination of knowledge across communities.

\section{CONFLICT OF INTEREST DISCLOSURES}

The author claims no conflicts of interest.

\section{REFERENCES}

1. Hwang ES, Hwang SY. Cellular NAD ${ }^{+}$level: a key determinant 
94 Jae-Young Lim

of mitochondrial quality and health. Ann Geriatr Med Res 2017;21:149-57.

2. Kwak JY, Kwon KS. Pharmacological interventions for treatment of sarcopenia: current status of drug development for sarcopenia. Ann Geriatr Med Res 2019;23:98-104.
Corresponding Author: Jae-Young Lim, MD, PhD

Department of Rehabilitation Medicine, Seoul National University Bundang Hospital, Seoul National University College of Medicine,

82 Gumi-ro 173beon-gil, Bundang-gu, Seongnam 13620, Korea

E-mail: drlim1@snu.ac.kr

ORCID: https://orcid.org/0000-0002-9454-0344

Received: September 26, 2019; Accepted: September 27, 2019 